\title{
Odorant Category Profile Selectivity of Olfactory Cortex Neurons
}

\author{
Ikue Yoshida and Kensaku Mori \\ Department of Physiology, Graduate School of Medicine, University of Tokyo, Bunkyo-ku, Tokyo 113-0033, Japan
}

The olfactory cortex receives converging axonal inputs from many mitral and tufted cells in the olfactory bulb. Recent studies indicate that single cortical neurons integrate signals from diverse odorants. However, there remains a basic question, namely, the signals from which kinds of odorants are integrated by the individual cortical neurons? The present study examined the possibility that some cortical neurons integrate signals from distinct component odorants of natural foods because individual foods produce a fixed combination of odorants. Previous psychophysical studies of core odorants emitted by fruits and vegetables suggest that the olfactory images of individual natural foods are basically characterized by the profile of structural and perceptual categories of food-born odorants. The single-unit spike responses of neurons in the dorsoposterior part of rat anterior piriform cortex to a panel of eight food-related categories of odorants were herein examined. The results showed that many cortical neurons in this region are tuned selectively to either a single category or a specific combination of distinct categories. The cortical neurons showed mixture facilitation and mixture inhibition when stimulated with mixtures of distinct categories, thus suggesting that olfactory circuits may play a role in enhancing the category-profile selectivity of individual neurons. These results indicate that signals from distinct categories of food-born odorants are integrated in these cortical neurons. This suggests that these cortical neurons detect the odorant-category profile of foods to distinguish distinct food odors.

Key words: olfactory cortex; food odor; odorant category; mixture facilitation; mixture inhibition; piriform cortex

\section{Introduction}

For most mammals, including humans, olfaction plays a critical role in object recognition, especially in food (Howard et al., 1968; Doty, 1986; Barnett, 2005; Laska et al., 2006; Shepherd, 2006). Individual foods emit hundreds of different odorants, and the odorant profiles are the defining elements of the distinct odor of individual foods (Goff and Klee, 2006). For the olfactory recognition of foods, therefore, olfactory system needs to integrate signals from the component odorants.

The early stages of mammalian olfactory system [olfactory epithelium and olfactory bulb (OB)] decompose the olfactory signals of individual objects into those of component odorants and code individual odorants based on their molecular features (Buck and Axel, 1991; Buck, 1996; Mori et al., 2006). Single principal neurons (mitral/tufted cells) in the OB receive olfactory sensory inputs in a single glomerulus that represent a single odorant receptor and send axonal outputs to many regions of the olfactory cortex (OC) (Shepherd et al., 2004).

Previous studies have shown that axonal projection of mitral/

Received April 13, 2007; revised July 10, 2007; accepted July 11, 2007.

This work was supported by Grants-in-Aid for Scientific Research on Priority Areas from the Ministry of Education, Culture, Sports, Science, and Technology and Grants-in-Aid for Scientific Research (S) from Japan Society for the Promotion of Science, Japan. We thank Y. Takahashi, K. M. Igarashi, and H. Matsumoto for their valuable technical advice, comments, and discussions. We also thank M. Yamaguchi, H. Nagao, H. Kashiwadani, S. Nagayama, H. Manabe, and Y. Tsuno for valuable discussions and members of Technical Research Center, T. Hasegawa Company (Kawasaki, Japan) for the gift of odorants and comments on food odors.

Correspondence should be addressed to Dr. Kensaku Mori, Department of Physiology, Graduate School of Medicine, University of Tokyo, 7-3-1 Hongo, Bunkyo-ku, Tokyo 113-0033, Japan. E-mail: moriken@m.u-tokyo.ac.jp. DOI:10.1523/JNEUROSCI.2720-07.2007

Copyright $\odot 2007$ Society for Neuroscience $\quad$ 0270-6474/07/279105-10\$15.00/0 tufted cells to the OC to be stereotypical, multiple, and overlapping (Zou et al., 2001; Neville and Haberly, 2004). Recent studies indicate that individual OC neurons receive converging inputs from distinct glomeruli (Zou et al., 2005; Zou and Buck, 2006). Single-cell recordings from the anterior olfactory nucleus $(\mathrm{AON})$, the most rostral region of the OC, suggest that individual AON neurons synaptically integrate inputs from several distinct mitral cells (Lei et al., 2006). These results strongly suggest that single cortical neurons integrate signals from diverse odorants. Does the cortical integration of signals from distinct odorants correlate with the specific combination of odorants that individual objects produce?

As a first step to address the above question, the responses of cortical neurons to food odors and their component odorants was examined. Although individual foods produce numerous different odorants, human sensory evaluation studies of odorants emitted from fruits and vegetables indicate that not all the compounds are necessary for food-odor recognition. Instead, only a specific subset of compounds (core odorants) is sufficient to mimic the basic olfactory image of a natural food (Jennings and Sevenants, 1964; Maarse, 1991). These studies, therefore, raised the possibility that brain processes the signals from the core odorants to form the basic olfactory image of a food. The published data of the core odorants of 33 natural foods were compiled (Japan Perfumery and Flavoring Association, 1989; Maarse, 1991; Arai, 2000; The Netherlands Organization for Applied Scientific Research, 2006) (Fig. 1). The data indicate that the core odorants can be classified into $\sim 14$ different categories based on their molecular structure and perceptual quality and suggest that the odor of individual foods can be characterized basically by 
either a single odorant category or a specific combination of the categories. This suggests that signals from specific odorant categories may thus be integrated by some neurons in the OC.

In the present study, the response selectivity of individual neurons in the anterior piriform cortex (aPC) was examined electrophysiologically using eight categories of core odorants of foods. The results indicate that individual OC neurons are tuned to specific combinations of odorant categories.

\section{Materials and Methods}

Animals. Experiments were performed on 31 male adult Wistar rats (7-12 weeks old; 170300 g; Japan SLC, Shizuoka, Japan). The animals were first anesthetized with medetomidine $(0.5 \mathrm{mg} / \mathrm{kg}$, i.p. $)$ and ketamine $(67.5 \mathrm{mg} / \mathrm{kg}$, i.p.) and then by urethane $(0.6 \mathrm{~g} / \mathrm{kg}$, i.p.). Adequate anesthesia was confirmed by the lack of a withdrawal response to hindlimb pinching. Additional doses of urethane were administered when necessary. Animals were placed in a stereotaxic frame that enabled us to fix the animal's head at any angle (custom made; Narishige, Tokyo, Japan) (Takahashi et al., 2004). Body temperature was maintained at $37.5^{\circ} \mathrm{C}$ using an animal blanket system (MK-900; Muromachi Kikai, Tokyo, Japan). Respiration was continuously monitored by measuring the chest movement with a strain gauge (TR-651T; Nihon Kohden, Tokyo, Japan) connected to a bridge module (AR-650H; Nihon Kohden) and an amplifier (AA-601H; Nihon Kohden). The heart rate and electroencephalogram (EEG) (recorded from the occipital cortex) were monitored routinely. All experiments were performed in accordance with the guidelines of the Physiological Society of Japan and the Animal Experiment Committee of the University of Tokyo.

Electrophysiology. Each animal was placed on its side in the stereotaxic frame. The skin covering the frontal bone was opened, the masseter muscle was detached from the skull, and the zygomatic arch was removed. Next, the bone overlying the lateral surface of piriform cortex (PC) was removed using a dental drill and fine forceps. To locate the recording area in the $\mathrm{aPC}$, a grid made of coordinate lines with an interval of $500 \mu \mathrm{m}$ was drawn on the photograph of the $\mathrm{PC}$ with the original point located at the intersection point between middle cerebral artery (MCA) and the dorsal edge of the lateral olfactory tract (LOT) (see Fig. $2 B, C$ ). This area was located at $500 \mu \mathrm{m}$ caudal to the MCA and $750 \mu \mathrm{m}$ dorsal from the original point, and extended $500 \mu \mathrm{m}$ in rostrocaudal axis and $750 \mu \mathrm{m}$ in dorsoventral axis (see Fig. $2 C$, green rectangle).

For extracellular single-unit recordings from aPC neurons, a glass micropipette (5-10 M $\Omega$ ) [filled with 2\% Chicago Sky Blue 6B (Tocris Bioscience, Bristol, UK) in $0.5 \mathrm{~m}$ sodium acetate) was inserted vertically into the area from the exposed surface of the PC. The recorded signals were amplified (AB-651J; Nihon Kohden), filtered using a bandpass filter (50 $\mathrm{Hz}$ to $3 \mathrm{kHz}$ ) (EW-610J; Nihon Kohden), and stored in a computer via an analog-to-digital converter (MICRO 1401; Cambridge Electronic Design, Cambridge, UK) using the Spike2 software program (Cambridge Electronic Design).

To identify the recorded position, Chicago Sky Blue 6B was iontophoretically injected into the recorded sites by applying a $5 \mu \mathrm{A}$ negative current, pulsed at $500 \mathrm{~ms} \mathrm{ON}, 500 \mathrm{~ms}$ OFF intervals for a period of $5 \mathrm{~min}$. After completing the experiments, the animals were deeply anesthetized and perfused first with saline and then with $4 \%$ paraformaldehyde (PFA) in $0.1 \mathrm{~m}$ phosphate buffer. After overnight fixation in $4 \%$ PFA, coronal sections of the brain ( $50 \mu \mathrm{m}$ in thickness) were obtained using a vibratome (DTK-3000; Dosaka, Kyoto, Japan). The sections were Nissl stained with $0.5 \%$ cresyl violet. The histological examination of the marked points indicated that all the recordings were obtained within the aPC.

For EEG recording, a stainless-steel screw was threaded into the bone above the occipital cortex ( $6 \mathrm{~mm}$ posterior from the bregma, $3.5 \mathrm{~mm}$ lateral from the midline). Another screw was then threaded into the bone above the cerebellum for reference. The signals were amplified, filtered (0.5-100 Hz) (EW-651J; Nihon Kohden), and stored in a computer via an analog-to-digital converter using the Spike2 software program.

Odor stimulation. For the stimulation with natural food odors, fresh fruits or vegetables were chopped into small pieces and placed in the test tube. Panel A included 21 natural and processed foods (apple, banana, grapefruit, tomato, cucumber, green tea, onion, Japanese radish, carrot, potato, coffee, shimeji mushroom, walnut, pumpkin seed, sweet potato, rice, rice bran, small dried sardines, sausage, cheese, ginger, and rat food pellet) and nine artificial food odors (apple-like, banana-like, grape-like, strawberry-like, cabbage-like, cucumber-like, garlic-like, and onion-like artificial odors). Panel B included 17 artificial food odors (strawberrylike, grape-like, watermelon-like, mango-like, melon-like, peach-like, raspberry-like, apple-like, cabbage-like, cucumber-like, sweet potatolike, shiitake mushroom-like, ginger-like, onion-like, tomato-like, and Japanese horseradish-like) and four essential oils (grapefruit, orange, celery, and carrot). The artificial food odors and essential oils were a kind gift from T. Hasegawa Company (Tokyo, Japan). Odor stimulation was performed by placing the opening of the odor-containing test tube at a distance of $1 \mathrm{~cm}$ from the animal's nostril.

For examining the odorant-category selectivity of the aPC neurons, eight categories of core odorants of natural foods were used (Fig. $2 A$ ): sulfides (Sul), methoxypyrazines (MPZ), C6C9 green compounds (C6C9), isothiocyanates (ITC), terpene hydrocarbons (tHC), esters, terpene alcohols $(\mathrm{tAl})$, and amines $\left(\mathrm{NH}_{2}\right)$. Each category is a mixture of 


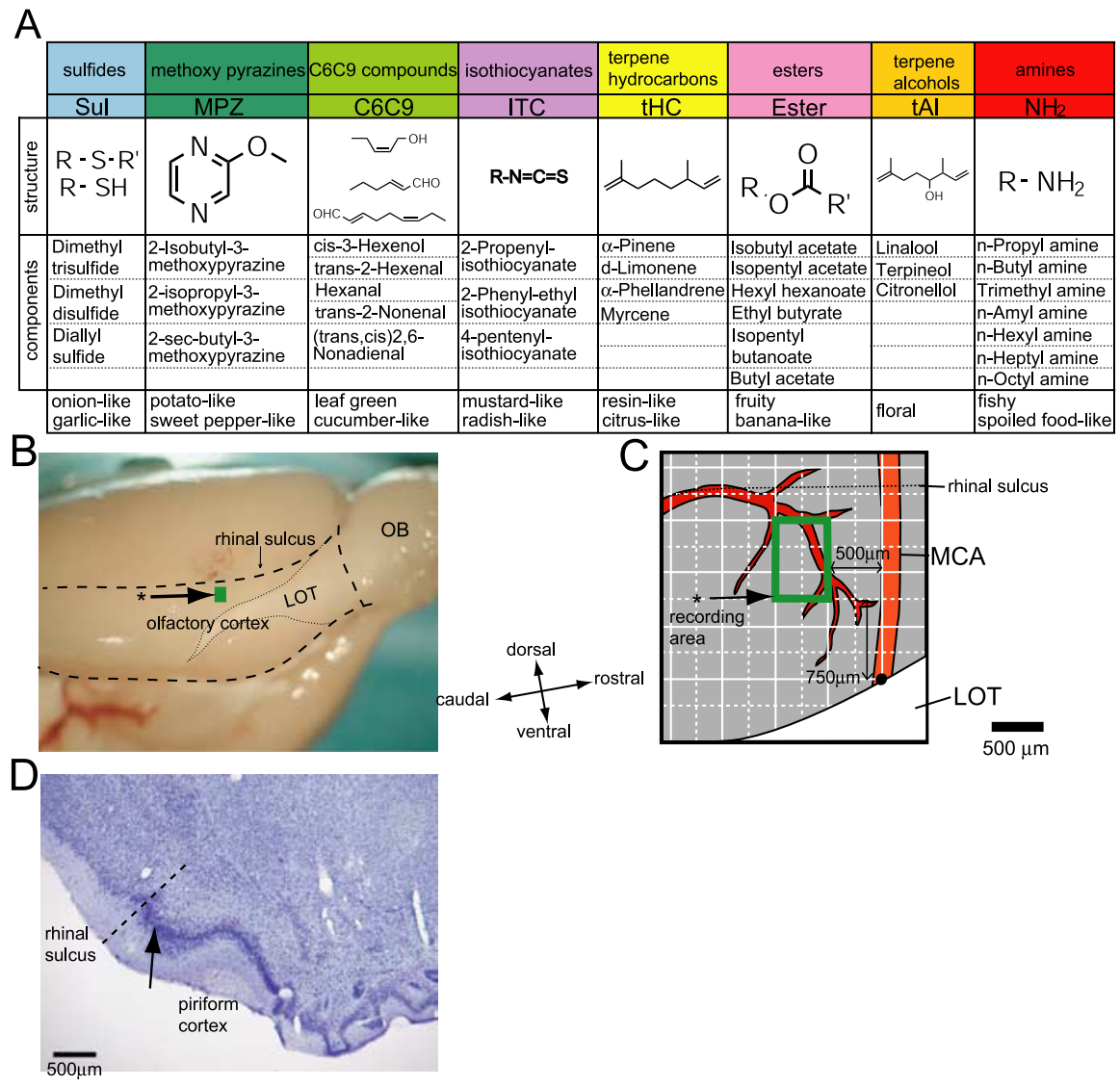

Figure 2. A panel of odorant categories used for the stimulation $(\boldsymbol{A})$ and the position of recording area in the aPC $(\boldsymbol{B}-\boldsymbol{D})$. $\boldsymbol{A}$, The panel consists of eight distinct categories (Sul, MPZ, $\mathrm{C}_{6} \mathrm{C} 9, \mathrm{ITC}, \mathrm{tHC}$, ester, $\mathrm{tAl}$, and $\mathrm{NH}_{2}$ ) of core odorants of natural foods. Each column shows (from top to bottom) the name of the category, abbreviation, molecular structure, component odorants in the mixture (3-7 odorants) used for the stimulation with each category, and the perceptual quality. $\boldsymbol{B}$, Ventrolateral view of the rat brain. The analysis focused on neurons located within the green rectangular area (an arrow with *). Boundaries of the olfactory cortex are shown by broken lines. LOT is indicated with a dotted line. C, A schematic diagram illustrating the dorsal part of the aPC that includes the green rectangular area in $\boldsymbol{B}$. With a reference to the intersection point (black dot) between MCA and the dorsalmost portion of the LOT, a $500 \times 500 \mu \mathrm{m}$ hypothetical grid was made on the surface of the olfactory cortex. Recording area is indicated by a green rectangle (an arrow with *). Recording microelectrodes were inserted into the green rectangular area under the visual guidance. $\boldsymbol{D}$, Coronal section of aPC stained with cresyl violet. The arrow indicates the recording point marked by the Chicago Sky Blue dye. Scale bar, $500 \mu \mathrm{m}$

three to seven different compounds. The components of Sul, MPZ, and $\mathrm{NH}_{2}$ were diluted in mineral oil to achieve a concentration of $2 \%(\mathrm{v} / \mathrm{v})$. The components of $\mathrm{C} 6 \mathrm{C} 9$, ITC, tHC, ester, and tAl were diluted to $5 \%$. Odorant stimulation was performed using a computer-controlled custom olfactometer, constructed of Teflon tubing, fittings, two-way solenoid valves (MTV-3-NM6; Takasago Electric, Nagoya, Japan), and 32channel digital input-output board (PCI-DIO-32HS; National Instruments, Austin, TX) controlled by custom software written by K. M. Igarashi, with LabVIEW (National Instruments). Saturated odor vapor was produced by flowing carbon-filtered pure air through disposable syringe filters (grass microfiber, $2.7 \mu \mathrm{m}$ pore size; Whatman, Springfield Mill, UK), which are loaded with $30 \mu \mathrm{l}$ of liquid odorant. The flow rates of the odorant-containing air streams were independently controlled by mass flow meters (Kofloc, Kyoto, Japan) and kept at $80 \mathrm{ml} / \mathrm{min}$. A carrier air stream was controlled by another mass flow meter to keep the total flow rate at $2000 \mathrm{ml} / \mathrm{min}$. Two-way microsolenoid valves controlled the timing of odor delivery. To maintain constant flow rates, the valves for blank filters (mineral oil) were opened when the valves for odor delivery were closed. Odor and carrier streams were mixed in a manifold downstream of all valves. An outlet for the odor stream was placed at a distance of $1 \mathrm{~cm}$ from the animal's nostril. Gas chromatographic examinations indicated the concentration of the components in the Sul mix at the animal's nostril to be $1.1 \mathrm{ppm}$ for dimethyl trisulfide, $0.1 \mathrm{ppm}$ for dipro- pyl disulfide, and $2.1 \mathrm{ppm}$ for diallyl sulfide. The concentration of the components in other mixtures was $2.8 \mathrm{ppm}$ for cis-3-hexenol (C6C9), $13.8 \mathrm{ppm}$ for isobutyl acetate (ester), and $0.08 \mathrm{ppm}$ for linalool (tAl). The duration for each odor application was $3 \mathrm{~s}$ with at least 30 s interstimulus intervals to avoid habituation (Wilson, 1998, 2001). For binary mixture stimulation, two microsolenoid valves that control the timing of two different odorant flows were opened simultaneously. This made it possible to keep the concentration of each category in the mixture the same as that of each category used for single category stimulation without a flow rate change. These odorants were obtained from Sigma (St. Louis, MO), Tokyo Chemical Industry (Tokyo, Japan), or Kanto Chemical (Tokyo, Japan).

Data analysis. An analysis of the spike responses was performed off-line using the Spike2 software program. Under urethane anesthesia, the neocortical EEG alternates between the slow-wave state (SWS) and the fastwave state (FWS) (Murakami et al., 2005). To determine the neocortical EEG state, the slowwave EEG $(0.5-2 \mathrm{~Hz})$ power and total EEG $(0.5-500 \mathrm{~Hz})$ power were measured for $10 \mathrm{~s}$ that includes the period of odor stimulation, and ratio of the two (slow-wave EEG power/total EEG power) was calculated. If this value was $>0.5$, it was designated SWS, and if it was $<0.3$, it was designated FWS. To minimize the influence of state-dependent sensory gating $(\mathrm{Mu}-$ rakami et al., 2005), all data of spike responses to odorants were obtained during FWS in the present study.

For individual neurons, responses to each category were recorded at least three times. The averaged firing rate was calculated at $100 \mathrm{~ms}$ bins. An excitatory response was defined as a significant increase in the averaged firing rate during the $3 \mathrm{~s}$ of odor presentation compared with the averaged firing rate during the $3 \mathrm{~s}$ of prestimulus period (1-4 s before the stimulus onset) ( $p<0.05$, Mann-Whitney $U$ test). An inhibitory response was defined as a significant decrease in the averaged firing rate during odor presentation compared with that during prestimulus period $(p<0.05$, Mann-Whitney $U$ test).

The magnitude of the odor-evoked response (MOR) was calculated by subtracting the number of spikes that occurred 1-4 s before the stimulus onset from the number of spikes that occurred during the stimulus period $(3 \mathrm{~s})$. For each odorant category, the averaged MOR (aMOR) was calculated. A response with $1.5 \leq$ aMOR $<5$ was defined as a weak response, $5 \leq \mathrm{aMOR}<10$ as a modest response, and $10 \leq \mathrm{aMOR}$ as a strong response.

The mixture facilitation was defined as those responses to the mixture whose aMOR was larger than the sum of aMORs of responses to each category. Mixture inhibition was defined as those responses to the mixture whose aMOR was lower than $50 \%$ of the aMOR of the larger response to each component category.

\section{Results}

\section{Responses of cortical neurons to food odors}

As a first step to examine the responses of cortical neurons to food odors, an area was arbitrary selected (Fig. $2 B, C$, green rectangle) within the dorsal part of the aPC and examined to determine whether that area contained neurons that respond to the odors of 
fruits and vegetables. The surface of the dorsal part of PC was surgically exposed, and the recording microelectrode was penetrated to the area of the aPC under visual guidance (Fig. $2 B, C$ ). The location of the recording was determined to be a $750 \times 500$ $\mu \mathrm{m}$ rectangular area $\sim 500 \mu \mathrm{m}$ caudal to the MCA and $\sim 750 \mu \mathrm{m}$ dorsal to the intersection between the MCA and the dorsal edge of the LOT (Fig. 2C). Single-unit discharges from neurons within the area of the aPC were recorded and examined to determine whether individual neurons in the area respond to food odors using panels of odorants that consist of arbitrary selected fruits and vegetables and artificial mixtures of odorants mimicking natural food odors (see Materials and Methods). Because the OC shows the state-dependent gating of sensory inputs under conditions of urethane anesthesia (Murakami et al., 2005), the responses to the odors were recorded only during the FWS (see Materials and Methods).

Among the 132 neurons recorded in the area of the aPC, 76 neurons $(58 \%)$ responded to at least one food odor in panels $\mathrm{A}$ and B (supplemental Fig. 3, available at www.jneurosci.org as supplemental material). The individual cortical neurons responded selectively to specific subsets of the food odors. For example, the cell in Figure $3 A$ responded selectively to six food odors (grape, raspberry, melon, strawberry, grapefruit, and crown daisy) among 21 food odors examined (cell 2 in supplemental Fig. 3B, available at www.jneurosci.org as supplemental material). The cell in Figure $3 B$ responded selectively to the watermelon odor among 19 food odors examined (cell 6 in supplemental Fig. 3B, available at www.jneurosci.org as supplemental material). The cell in Figure $3 C$ showed a strong response to carrot odor and modest response to apple and strawberry odors but did not respond to the other 17 food odors (cell 10 in supplemental Fig. 3A, available at www.jneurosci.org as supplemental material).

Because many neurons in the area showed robust responses to specific subsets of food odors, it appeared that the foodresponsive neurons would participate in the integration of signals from diverse food-born odorants. Which combination of component odorants of food do the individual cortical neurons respond to?

Natural foods typically produce hundreds of different odorants and contain many unknown odorants. Moreover, they show a profound change in their odor attributable to seasons, ripeness, and spoilage (Menager et al., 2004). Therefore, natural foods are not suitable as reproducible and reliable stimuli to examine the response selectivity of OC neurons. To prepare the panel of the stimulus odorants composed of the known components of natural foods, a database of component odorants of fruits and vegetables was consulted (Japan Perfumery and Flavoring Association, 1989; Maarse, 1991; Arai, 2000; The Netherlands Organization for Applied Scientific Research, 2006). Psychophysical studies of food odors indicate that only a specific subset of the emitted compounds are sufficient to mimic the basic olfactory image of a natural food (Jennings and Sevenants, 1964; Maarse, 1991). Some compounds (character impact compounds) individually possess the odor suggestive of a particular food. Other compounds (contributory flavor compounds) do not individually have the odor of a particular food but contribute odor characteristics such as fruitiness or greenness. In this report, the sum of the character impact compounds and contributory flavor compounds will be referred to as the "core odorants." The core odorants of 33 different foods are listed in Figure 1 (and supplemental Figs. 1, 2, available at www.jneurosci.org as supplemental material). The data in Figure 1 indicate that core odorants
A
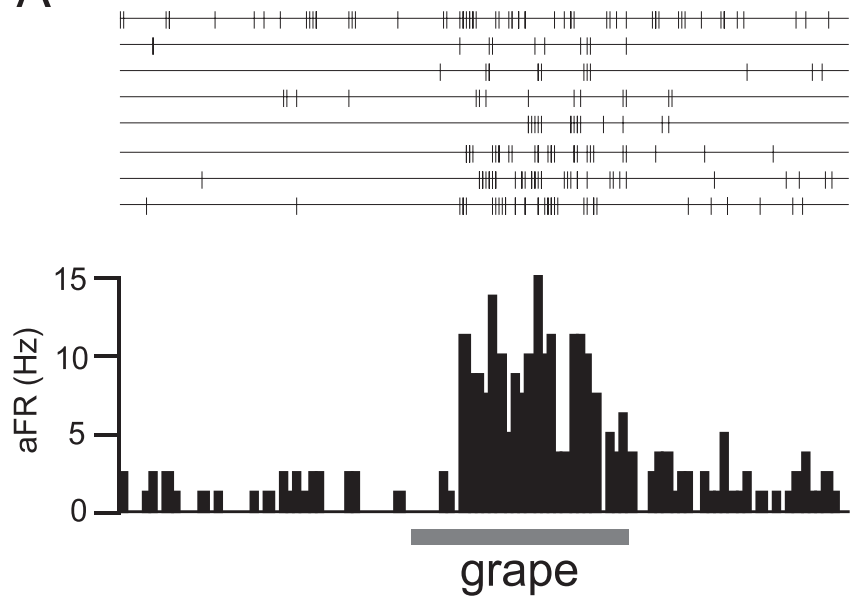

B
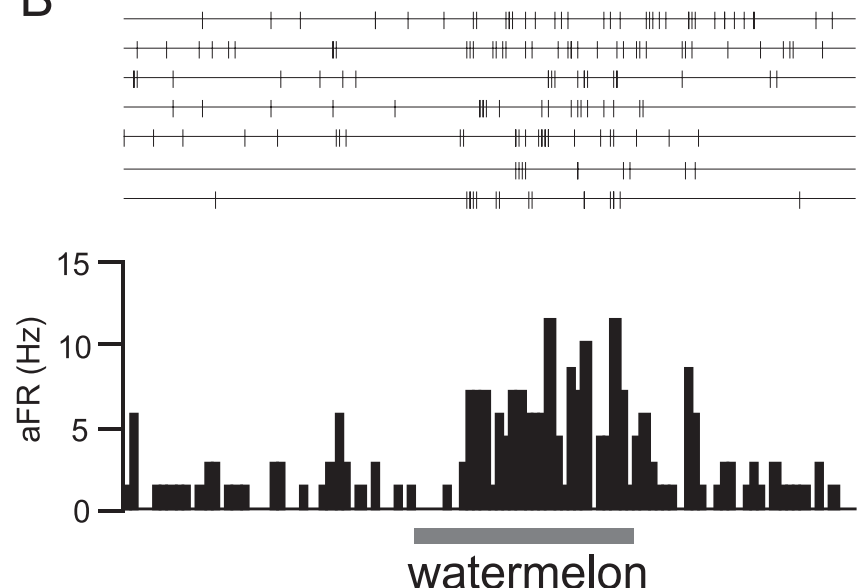

C
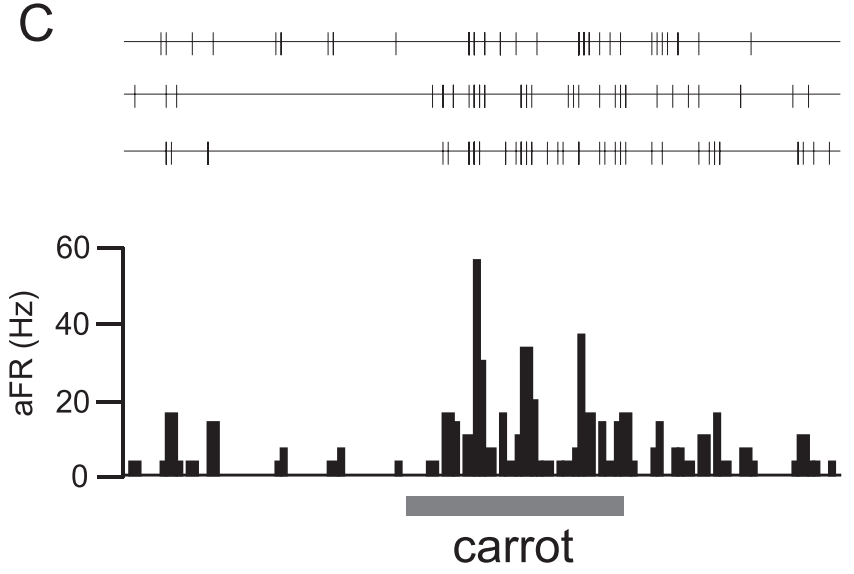

Figure 3. Olfactory cortex neurons in the area of aPC respond to food odors. $A$, Spike traces and peristimulus time histogram of a neuron in the area of the aPC, showing strong responses to the mixture of odorants that mimics the grape odor. This neuron was found in layer 3. $\boldsymbol{B}$, Responses of another neuron (layer 2) to the artificial odor of watermelon. $\boldsymbol{C}$, Responses of another neuron (layer 3) to the odor of raw carrot. aFR, Averaged firing rate. The bar under the histogram indicates the period of odor stimulation (3s).

can be classified into $\sim 14$ odorant categories based on their molecular structure and perceptual quality. The odorant categories include sulfides, alcohols, methoxypyrazines, so called 6-carbon and 9-carbon green-odor compounds, aldehydes, ketones, isothiocyanates, terpene hydrocarbons, esters, terpene alcohols, alkylamines, acids, lactones, and phenol and its derivatives (phe- 


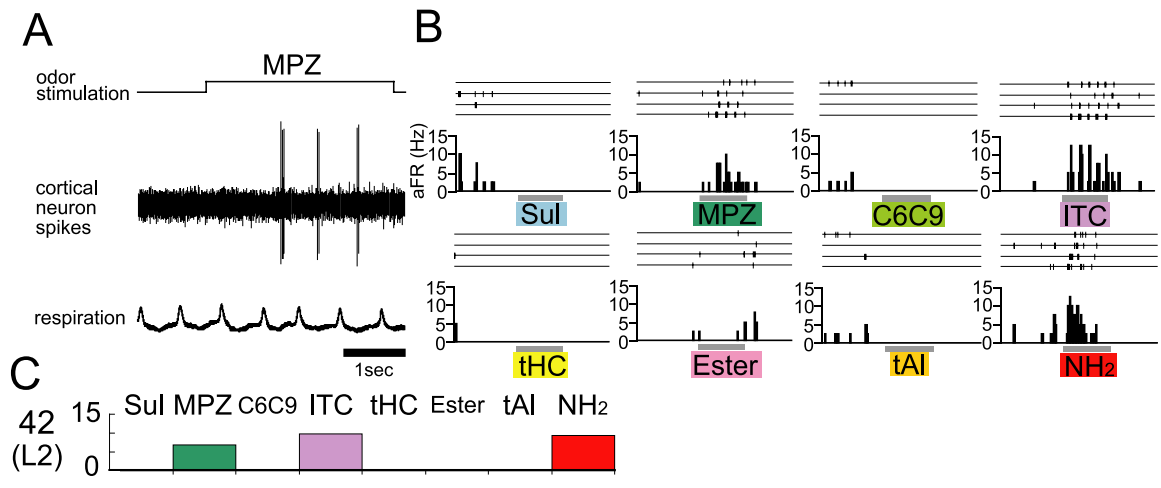

Figure 4. Response selectivity of a neuron in the area among the eight odorant categories examined. $\boldsymbol{A}$, Spike responses of an aPC neuron to MPZ (middle trace). The top trace indicates the period of MPZ stimulation (upward deflection, $3 \mathrm{~s}$ ). The bottom trace shows the respiration pattern. Upward deflection indicates inhalation. Scale bar, 1 s. B, Spike traces and peristimulus time histogram of responses of the same neuron to the stimulation with individual odorant categories. This neuron showed strong responses to MPZ, ITC, and $\mathrm{NH}_{2}$ but showed almost no response to the other categories. $\boldsymbol{C}$, Schematic representation of the odorant-category selectivity of this neuron (neuron 42). Excitatory responses were shown by the upward columns. This neuron was found in layer 2.

nol). The data suggest that the odors of individual natural foods can be characterized basically by either a single odorant category or specific combination of them. Based on these data, it is possible that individual OC neurons would be tuned to distinct odorant-category profiles. Therefore, a panel of stimulus odors consisting of eight categories of core odorants of foods (Sul, MPZ, C6C9, ITC, tHC, ester, tAl, and $\mathrm{NH}_{2}$ ) (Fig. $2 \mathrm{~A}$ ) was prepared. A mixture of three to seven different compounds was used for stimulation with each category (Fig. 2A).

\section{Odorant-category selectivity of individual neurons in the olfactory cortex}

Using the panel of eight odorant categories, the response selectivity of the 92 neurons in the area of aPC was examined in detail. The recordings of single-cell spike discharges typically lasted from 20 to $120 \mathrm{~min}$, during which time the responses to each category of odorants were examined at least three times. A majority of neurons (70 cells, 76\%) responded to at least one category among the eight categories. Fifty-seven cells showed excitatory responses (increased spike discharges) to at least one category, thus indicating a good efficacy of the panel of stimulus odorant categories to activate neurons in the area of the aPC. The remaining 13 cells showed only the inhibitory responses (decreased spike discharges).

The neurons in the area of the aPC responded selectively to a single category or to specific subsets of the eight categories. Figure 4 exemplifies the response selectivity of individual neurons. This cell responded to MPZ with burst discharges that were phase locked to the respiration (Fig. $4 A$ ). In addition, this cell was activated by ITC and $\mathrm{NH}_{2}$ (Fig. $4 \mathrm{~B}$ ). Other odorant categories did not elicit a significant response. Therefore, this cell is tuned selectively to the three categories, MPZ, ITC, and $\mathrm{NH}_{2}$, as shown schematically in Figure 4C.

The category selectivity of individual neurons in the area is summarized schematically in Figure 5. In this diagram, an individual neuron is shown by a horizontal thick line with a neuron number and the recorded layer. Excitatory responses are shown by upward columns, whereas inhibitory responses by downward hatched columns. Among the 57 neurons that showed excitatory responses, 24 neurons (42\%) showed excitatory responses selectively to a single category (single category neurons, left box).
Interestingly, there were a variety of single category neurons in terms of category selectivity; six cells (25\%) were selective to the category of Sul, five cells $(20 \%)$ to MPZ, four cells (16\%) to C6C9, three cells $(12 \%)$ to tHC, two cells $(8 \%)$ to ester, three cells (12\%) to tAl, and one cell (4\%) to $\mathrm{NH}_{2}$.

Thirty-three neurons (58\%) showed excitatory responses to more than two categories of odorants (multiple category neurons, middle and right top boxes), thus suggesting that these neurons receive converging inputs from distinct categories of food-born odorants. Eleven neurons were selectively activated by two categories, 12 neurons by three categories, four neurons by four categories, two neurons by five categories, and three neurons by six categories. One neuron (neuron 57) was exceptional in that it was activated by all eight categories. For the majority of the multiple category neurons, the most adequate odorant category was defined as indicated by an asterisk in Figure 5. The most adequate category varied across different multiple category neurons.

The recorded neurons were located at layers 1,2, and 3 of the aPC. Among 57 neurons that showed excitatory responses, 28 neurons were recorded at layers 1 and 2 (superficial layers), and 29 neurons were located at layer 3 (deep layer). Single category neurons were found in both superficial (11 cells) and deep (13 cells) layers. Multiple category neurons were distributed in both superficial (17 cells) and deep (16 cells) layers. As described in previous studies (Wilson, 1998; Litaudon et al., 2003; Rennaker et al., 2007), in all the recorded neurons, odorant-induced spike discharges were phase locked to the respiration cycle. During the period of odor stimulation ( $3 \mathrm{~s}$ ), animals typically showed inhalation four to eight times. In $81 \%$ of the neurons, the respiration phase-locked spike discharges were maintained during the stimulation (Fig. 4). The remaining 19\% of neurons, however, showed a rapid adaptation to the odorant stimulation and responded to only a few inhalations.

Figure 5 indicates that the multiple category neurons varied widely from neuron to neuron in terms of category-profile selectivity. Cell 27, for example, showed excitatory responses selectively to Sul and ITC, whereas cell 36 responded selectively to Sul, MPZ, and ester. The wide repertoire of the category-profile selectivity among recorded neurons suggests the importance of specific combinations of odorant categories for activating the individual neurons in the aPC.

To estimate which pairs of the eight odorant categories tended to be combined by individual neurons, the number of cells that showed excitatory responses to both of the two categories for each binary combination of the eight odorant categories (Fig. 6) were counted, based on the data shown in Figure 5. Because one cell (Fig. 5, cell 57) responded to all eight categories, this cell was therefore excluded from the counting. The results indicate that all of the binary combinations were observed in the population of the recorded neurons. The most frequent combinations observed were cells that responded to both ester and C6C9 (eight cells), the cells that responded to tHC and C6C9 (eight cells), and the cells that responded to $\mathrm{C} 6 \mathrm{C} 9$ and Sul (eight cells). The second most frequently encountered cells were those that responded to ester and Sul (seven cells), those that responded to tHC and MPZ 
(seven cells), and those that responded to ester and MPZ (seven cells). For the ternary combination of the eight categories, the cells that responded to $\mathrm{C} 6 \mathrm{C} 9, \mathrm{tHC}$, and ester (five cells) were the most frequently recorded.

\section{Responses of olfactory cortex neurons to the binary mixtures of the odorant categories}

Because food odors are mixtures of distinct odorant categories and the individual neurons in the area responded selectively to the odorant-category profiles, the response selectivity of the OC neurons to binary mixtures of the eight categories of core odorants was examined. An olfactometer specifically designed for the stimulation with odorant mixtures was used, so that the concentration of each component category in the mixture is same as that of each category used for component stimulation (see Materials and Methods). Because previous studies demonstrated the mixture facilitation in OC neurons (Wilson, 2003; Kadohisa and Wilson, 2006; Lei et al., 2006; Zou and Buck, 2006), it is possible that, if some neurons in the OC function as a category-combination detector responding maximally to a specific mixture of distinct odorant categories, these neurons would respond more strongly to the binary mixture than to each component category (mixture addition) or would even show an excitatory response to the mixture that exceeds the sum of the responses to each component category (mixture facilitation).

Figure 7 shows two examples of OC neurons that showed mixture facilitation to the binary mixture of odorant categories. The cell in Figure $7 A$ showed a weak inhibitory response to ester but also showed a negligible or very small response to ITC. In a striking contrast, this cell showed a clear response to the binary mixture of ester and ITC. Another neuron (Fig. 7B) showed only a small response to each of Sul and C6C9, whereas its response to the mixture of Sul and $\mathrm{C6C} 9$ exceeded the sum of the responses to each component category. Among the 41 cells examined, mixture facilitation was observed in 24 neurons (59\%) in the area of the aPC. Neurons that showed mixture facilitation were found in both superficial (11 cells) and deep (13 cells) layers.

The OC contains a variety of inhibitory interneurons (Neville and Haberly, 2004), thus suggesting that odorant stimulation induces inhibitory responses in cortical neurons in addition to the excitatory responses. Figure 5 illustrates that many neurons in the area showed excitatory responses to a subset of the categories and inhibitory responses to other categories (Fig. 5, cells 4-6, 10, $11,21-23,29,30,33-35,44$, and 47). With extracellular singleunit recordings, however, the odorant-induced inhibitory responses could only be observed when the recorded neurons showed spontaneous discharges. To examine the effect of inhibition more clearly, the ability of individual neurons to show mixture inhibition (Wilson, 2003; Kadohisa and Wilson, 2006) to the binary mixtures of odorant categories was thus examined.

Figure $8 \mathrm{~A}$ shows an example of mixture inhibition. This cell showed a clear excitatory response to ITC (middle) but showed no response to Sul (left). When the olfactory epithelium was stimulated with a mixture of Sul and ITC, this cell did not show any response (right), thus indicating that the ITC-induced response was suppressed by the copresence of Sul. This suggests that both the presence of ITC and the absence of Sul are necessary to activate this neuron. The mixture inhibition thus enables the cortical neuron to distinguish "ITC without Sul" from "ITC with Sul". The excitatory responses to one category were frequently suppressed by the mixture with another category that itself 


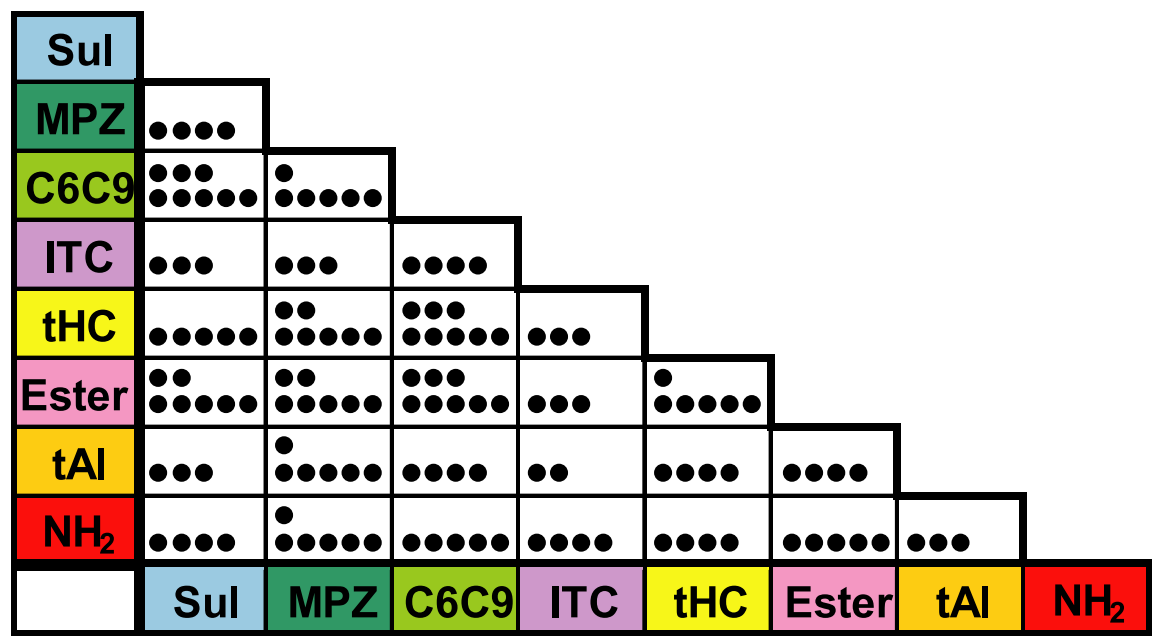

Figure 6. A matrix representation of the number of recorded neurons that received excitatory inputs from a specific binary combination of the eight categories. The number of dots in each rectangle indicates the number of aPC neurons that responded to both the category shown at the horizontal axis (bottom row) and the category shown at the vertical axis (leftmost column). This matrix was based on the data of 32 multiple category neurons (excluding neuron 57) in Figure 5. Note that all of the binary combinations of the eight categories were observed in the recorded neurons.

A

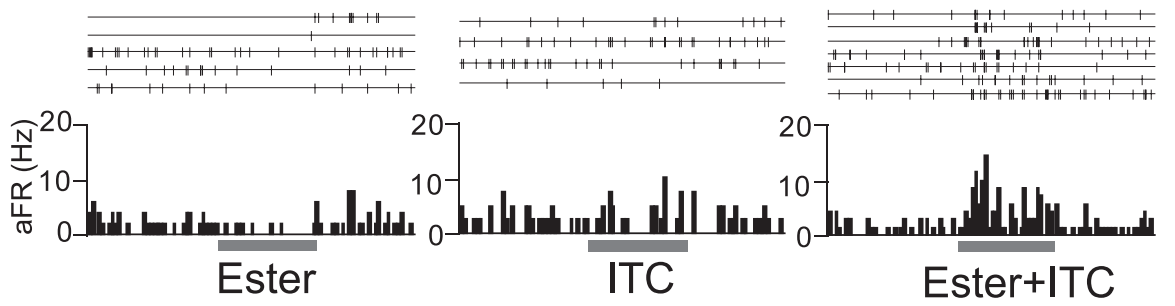

B

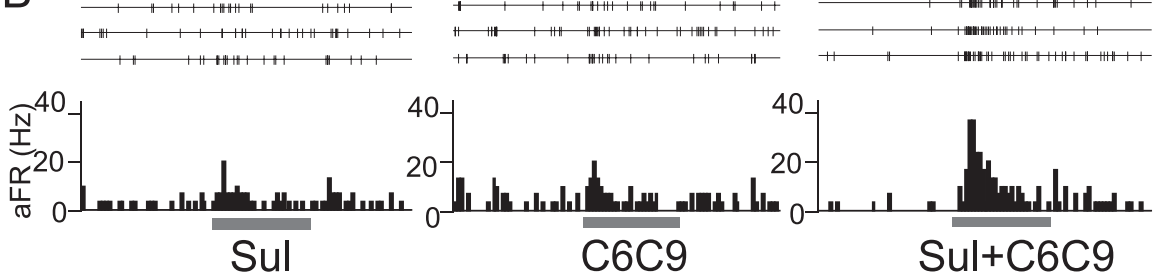

Figure 7. Mixture facilitation of odor-evoked responses of aPC neurons. $A$, Spike traces and peristimulus time histogram of a neuron in the area of the aPC. This neuron (layer 2) showed small inhibitory responses to ester (left histogram) but showed no significant response to ITC (middle). When stimulated with the mixture of these categories, however, this neuron showed strong excitatory responses (right). $\boldsymbol{B}$, Another aPC neuron (layer 3) showing only weak excitatory responses to Sul (left) and C6C9 (middle). This neuron showed a robust response when stimulated with the mixture of Sul and C6C9. aFR, Averaged firing rate. The bar under the histogram indicates the period of odor stimulation (3 s).

evoked no excitatory or inhibitory response. This simple type of mixture inhibition was observed in most of the cortical neurons examined ( $84 \%, 26$ cells of 31 cells examined): in seven neurons among eight single category neurons and in 19 neurons among 23 multiple category neurons. Neurons that showed the simple type of mixture inhibition was found in both superficial ( 16 cells) and deep ( 10 cells) layers.

In addition to the simple type of mixture inhibition, multiple category neurons showed complex type of mixture inhibition (Fig. $8 B$ ). The neuron in Figure $8 B$ responded robustly to Sul (left) and ester (middle) but only showed a small or negligible response to the mixture of them (right). Therefore, the signals from Sul and those from ester interacted with each other so that this cell could not be activated effectively by the mixture of these categories. This type of mixture inhibition enables the cortical neuron to distinguish "Sul without ester" and "ester without Sul" from "ester and Sul". This complex type of mixture inhibition was observed in 16 neurons $(70 \%)$ of 23 multiple category neurons examined. Among these 16 neurons, eight cells were recorded at the superficial layers, and the remaining eight cells were located at the deep layer.

In 41 neurons, the responses to all the binary mixtures of the eight categories ( 28 different combinations) were systematically compared with the responses to individual categories. Many single category neurons showed a category-combination selectivity of mixture inhibition, i.e., they showed mixture inhibition when the category effective in activating the recorded cell was mixed with specific categories, but they did not show mixture inhibition when mixed with the other categories. For example, the cell in Figure $9 A$ showed an excitatory response to MPZ. As shown in the right column of Figure $9 A$, the MPZinduced response was effectively suppressed when MPZ was mixed with Sul, C6C9, tHC, ester, or $\mathrm{NH}_{2}$. In contrast, the mixture with ITC or tAl had little effect on the MPZ-induced response. Therefore, not only the presence of the MPZ but also the absence of Sul, C6C9, tHC, ester, and $\mathrm{NH}_{2}$ were necessary to activate this neuron. Figure $9 B$ schematically shows the category-combination selectivity of mixture inhibition of this neuron.

Figure 9, $C$ and $D$, shows another example of selective mixture inhibition. This cell responded robustly to Sul, and the Sulinduced response was suppressed when Sul was mixed with either MPZ or ester. In contrast, the copresence of C6C9, ITC, $\mathrm{tHC}, \mathrm{tAl}$, or $\mathrm{NH}_{2}$ caused little change in the Sul-induced response. Therefore, the presence of Sul and absence of MPZ and ester were both necessary to effectively activate this neuron. The categorycombination-selective mixture inhibition was observed in six neurons among eight single category neurons examined.

Multiple category neurons often showed both the simple type and complex type of mixture inhibitions. The cell in Figure 9, E and $F$, responded strongly to $\mathrm{NH}_{2}$ (left column in $E$ ) and MPZ and ITC (middle column). When $\mathrm{NH}_{2}$ and ITC were mixed, the response of this cell to the mixture was smaller than the responses to each component. A similar complex type of mixture inhibition was observed with the combination of $\mathrm{NH}_{2}$ and $\mathrm{MPZ}$ and with the combination of MPZ and ITC (data not shown). This cell also showed the simple type of mixture inhibition. The $\mathrm{NH}_{2}$-induced excitatory response was inhibited by the copresence of Sul, C6C9, ester, or tAl, all categories that did not evoke either excitatory or inhibitory responses. However, the $\mathrm{NH}_{2}$ response was not inhibited by tHC (Fig. 9E). MPZ response and ITC response of this 
neuron were inhibited by the copresence of Sul, C6C9, tHC, ester, or tAl (data not shown). This cortical neuron thus is tuned to " $\mathrm{NH}_{2}$ without other categories (except tHC)," "ITC without any other categories," and "MPZ without any other categories" and thus can distinguish them from virtually all the binary combinations of the eight categories.

Present results demonstrated that many aPC neurons that showed a category-combination selectivity of simple type of mixture inhibition. Similarly, we observed the category-combination selectivity of complex type of mixture inhibition in 10 cells among 11 cells that showed the complex type of mixture inhibition (data not shown). In the case of mixture facilitation, the category-combination selectivity was observed in all neurons $(n=$ 24) examined.

\section{Discussion}

Food-odor perception is of life-and-death importance for wild animals. What is the neural basis for the detection and discrimination of natural food odors? How are the signals of diverse food-born odorants processed in the OC? To elucidate the manner of processing foododor information in the OC, the response selectivity of aPC neurons to the eight odorant categories of core odorants of fruits and vegetables were analyzed. The results indicate that (1) individual neurons in the area of the aPC are tuned to specific profiles of the odorant categories, and (2) category-profile selectivity of individual neurons was enhanced by mixture facilitation and/or mixture inhibition.

\section{Odorant-category profile selectivity of individual neurons in the aPC}

Based on the data of odorants emitted from fruits and vegetables, the core odorants were classified into 14 different categories. One hypothesis is that individual cortical neurons might integrate signals from distinct odorant categories. Using the panel of eight different odorant categories, the response selectivity of individual cortical neurons in the area of the aPC was examined.

Consistent with the above hypotheses, the present results showed that individual neurons in the area of the aPC are tuned to either a single category or a combination of them (Fig. 5). Surprisingly, the neurons in the area showed a wide variety of category-profile selectivity (Figs. 5, 6). Does the odorantcategory profile selectivity of individual cortical neurons correlate with the odorant-category profile of the individual natural foods? The present results suggest a possible correlation. For example, some neurons showed excitatory responses selectively to the Sul category and this selectivity might correlate with the fact that odors of onion, garlic, shallot, and asparagus are basically characterized by the Sul category (Fig. 1). Neurons that showed excitatory response selectively to MPZ category might correlate with the MPZ-selective profile of core odorants of beet, potato, and lettuce odors (Fig. 1). Several neurons were observed that showed excitatory responses to both the C6C9 category and the ester category (Figs. 5, 6). The odorant-category selectivity of these neurons might correlate with the fact that odors of apple, banana, and melon are characterized basically by the combina- tion of C6C9 category and ester category (Fig. 1). The wide variety of category-profile selectivity of the aPC neurons raises the possibility that neuronal circuits in the aPC might participate in the discrimination of a variety of odorant-category profiles that diverse foods produce. We thus speculate that neurons in the area of the aPC are involved in the detection of and discrimination among diverse food odors.

\section{Responses of cortical neurons to binary mixture of odorant categories}

Previous studies demonstrated mixture facilitation of odorevoked responses in OC neurons using the methods of extracellular single-unit recordings (Wilson, 2003; Kadohisa and Wilson, 2006), intracellular recordings (Lei et al., 2006), and cellular compartment analysis of temporal activity by fluorescence in situ hybridization (Zou and Buck, 2006). We extended these studies using a systematic panel of odorant categories and all their binary combinations and found the category-combination selectivity of mixture facilitation. These results (Fig. 7) indicate that these neurons function as detectors of specific combinations of odorant categories. These neurons might therefore be useful for detecting fruits or vegetables that contain a specific combination of odorant categories.

In addition, mixture inhibition can enhance the function of a combination detector. Using four different odors, Kadohisa and Wilson (2006) showed that $\sim 67 \%$ of aPC neurons exhibit mixture suppression. In line with previous findings, we observed mixture inhibition in $73 \%$ of aPC neurons. The present study systematically examined the responses of cortical neurons to all of the 28 binary combinations of the eight odorant categories and found a category-combination selectivity of mixture inhibition (Fig. 9). The category-combination selectivity was observed for both simple type and complex type of mixture inhibition. The observation of the simple type of mixture inhibition (Figs. $8 \mathrm{~A}$, $9 A-D$ ) indicates that the presence of a specific combination of categories and the absence of other specific categories are both necessary to activate individual neurons. This suggests that mixture inhibition enhances the selective tuning of individual neu- 


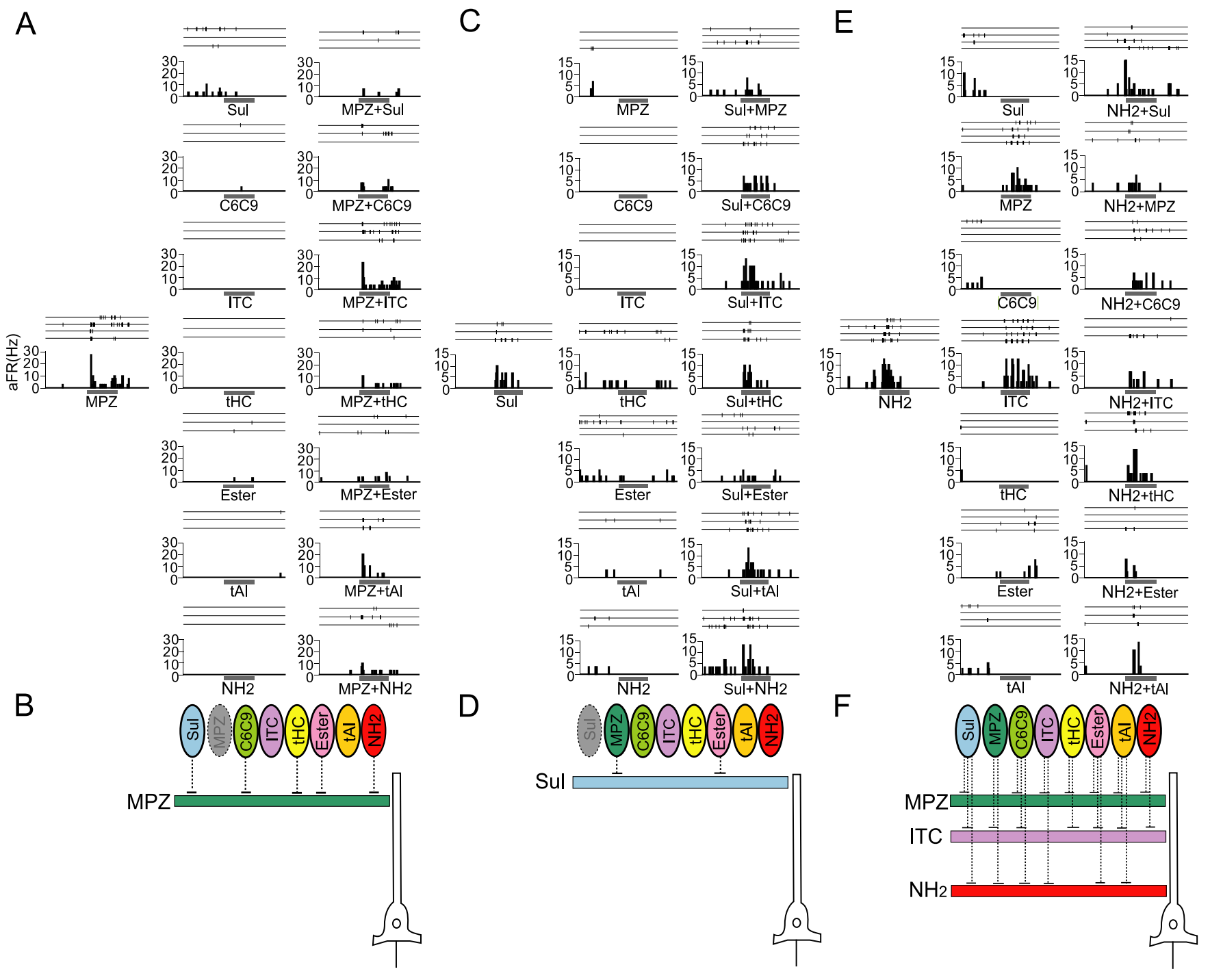

Figure 9. Category-combination selectivity of mixture inhibition of aPC neurons. A, Spike traces and peristimulus time histogram of a neuron in the area of the aPC (layer 1). This neuron showed excitatory responses to MPZ (left column) but also showed no significant response to other seven categories (middle column). The excitatory response to MPZ was suppressed when mixed with Sul, $\mathrm{C} 6 \mathrm{C} 9$, $\mathrm{tHC}$, ester or $\mathrm{NH}_{2}$. However, the mixture with ITC or tAl did not effectively suppress the MPZ-evoked response. $\boldsymbol{B}$, A schematic diagram of the category-combination selectivity of mixture inhibition of the neuron shown in $A$. The horizontal bar with color indicates the category (MPZ) that evoked excitatory response in this neuron. The excitatory response to MPZ was suppressed when mixed with the categories that are linked by the dotted line (shown by ellipses with color). Mixture with categories shown by ellipses without the dotted line did not suppress the MPZ-induced response. $\boldsymbol{C}$, Another neuron (layer 3) showing excitatory responses to Sul. The remaining seven categories showed no significant response. The excitatory response to Sul was suppressed when mixed with MPZ or ester. However, mixture with other categories did not suppress the Sul-evoked response. $\boldsymbol{D}$, A schematic diagram of the category-combination selectivity of mixture inhibition of the neuron in $\boldsymbol{C}$. $\boldsymbol{E}$, Another neuron (layer 2) showing the complex type of mixture inhibition. This neuron showed excitatory response to $\mathrm{NH}_{2}, \mathrm{MPZ}$, and ITC. Mixture inhibition was observed when two of them were mixed $\left[\mathrm{NH}_{2}+\mathrm{MPZ}\right.$, NH $\mathrm{N}_{2}+$ ITC (right column) and ITC + MPZ (data not shown)]. $F$, Schematic diagram of the complex type of mixture inhibition of the neuron in $E$. The MPZ-induced response (horizontal bar with MPZ) was suppressed when MPZ was mixed with Sul, $66 \mathrm{C}$, ITC, tHC, ester, tAl, or NH 2 . ITC-induced response (horizontal bar with ITC) was suppressed when ITC was mixed with Sul, MPZ, $\mathrm{C6C}_{\mathrm{C}}$, tHC, ester, tAl, or $\mathrm{NH}_{2}$. The $\mathrm{NH}_{2}$-induced response (horizontal bar with $\mathrm{NH}_{2}$ ) was suppressed when $\mathrm{NH}_{2}$ was mixed with Sul, MPZ, C6C9, ITC, ester, or tAl. aFR, Averaged firing rate. The bar under the histogram indicates the period of odor stimulation ( $3 \mathrm{~s}$ ).

rons to specific profile of odorant categories by suppressing their responses when other categories are intermixed. Mixture inhibition may thus enable the olfactory system to discriminate the food odors composed of categories $X$ and $Y$ from those composed of categories $X, Y$, and $Z$.

Because individuals in a species (e.g., mice, rats, and even human) are thought to produce unique odorant profiles and thus have their own body odor (Beauchamp and Yamazaki, 2003; Penn et al., 2007), the odorant profiles might be the defining elements for the olfactory recognition of individuals. This raises the possibility that odorant profile detection is a common and basic function of OC neurons for object recognition.

Although the present study provides evidence for the category-profile selectivity of individual neurons in the OC, it remains necessary to expand the panel of stimulus odorant categories of food-born odorants to include those not examined in the present study (alcohols, aldehydes, ketones, acids, lactones, and phenols) to determine in more detail the category-profile selectivity of individual neurons. It is also necessary to examine the effect of change in the concentration ratio of component categories on the response selectivity of individual cortical neurons because each food typically emits a mixture of categories with relatively fixed proportion of component categories (The Netherlands Organization for Applied Scientific Research, 2006). Furthermore, fruit and vegetable maturation is known to accompany a profound change in their odor and in relative proportion 
of the odorant categories (Menager et al., 2004). With the increasing maturity of strawberry, for example, acids and esters increase, whereas C6C9 decreases. Therefore, OC neurons might detect not only the odorant-category profile but also the ratio of distinct odorant categories.

\section{Possible neuronal circuits responsible for the category-profile selectivity of individual cortical neurons}

The presence of many multiple category neurons in the area of the aPC suggests that these cortical neurons receive convergence of signals from distinct mitral cells. This has also been observed in individual neurons in the AON (Lei et al., 2006). The convergence of signals from different categories might be attributable to the monosynaptic convergence of afferent fibers from distinct mitral cells. Alternatively, the convergence might be mediated polysynaptically via association fibers of the pyramidal cells in the OC (Neville and Haberly, 2004) or the combination of afferent and association fibers.

The present results showed that neurons with a variety of category-profile selectivity are found in a small area of the aPC. These findings correlate with the anatomical evidence that a small area of the aPC receives converging axonal inputs from many different regions of the OB (Haberly and Price, 1977; Skeen and Hall, 1977; Scott et al., 1980; Luskin and Price, 1982). It should be underscored that, although the area of the aPC receives converging inputs from all the eight categories of core odorants, the individual neurons are tuned to specific subsets of these categories. These results raise an intriguing question regarding how the odorant-category profiles are spatially represented in the aPC.

In summary, this study revealed that individual neurons in the aPC responded to odors of fruits and vegetables. Examinations with a panel of eight categories of the core odorants of natural foods indicated that individual neurons responded selectively to a single category or to a specific combination of categories. These results suggest that neuronal circuits in the aPC detect and discriminate distinct category profiles of core odorants of foods and thus participate in the detection of and discrimination among distinct food odors.

\section{References}

Arai S (2000) New encyclopedia of odorants (in Japanese). Tokyo: Asakura. Barnett SA (2005) Ecology. In: The behavior of the laboratory rat. A handbook with tests (Whishaw I, Kolb B, eds), pp 15-24. New York: Oxford UP.

Beauchamp GK, Yamazaki K (2003) Chemical signalling in mice. Biochem Soc Trans 31:147-151.

Buck L, Axel R (1991) A novel multigene family may encode odorant receptors: a molecular basis for odor recognition. Cell 65:175-187.

Buck LB (1996) Information coding in the vertebrate olfactory system. Annu Rev Neurosci 19:517-544.

Doty RL (1986) Odor-guided behavior in mammals. Experientia 42:257-271.

Goff SA, Klee HJ (2006) Plant volatile compounds: sensory cues for health and nutritional value? Science 311:815-819.

Haberly LB, Price JL (1977) The axonal projection patterns of the mitral and tufted cells of the olfactory bulb in the rat. Brain Res 129:152-157.

Howard WE, Marsh RE, Cole RE (1968) Food detection by deer mice using olfactory rather than visual cues. Anim Behav 16:13-17.

Japan Perfumery and Flavoring Association (1989) Encyclopedia of flavors (in Japanese). Tokyo: Asakura.
Jennings WG, Sevenants MR (1964) Volatile esters of bartlett pear III. J Food Sci 29:158-163.

Kadohisa M, Wilson DA (2006) Olfactory cortical adaptation facilitates detection of odors against background. J Neurophysiol 95:1888-1896.

Laska M, Freist P, Krause S (2006) Which senses play a role in nonhuman primate food selection? A comparison between squirrel monkeys and spider monkeys. Am J Primatol 69:1-13.

Lei H, Mooney R, Katz LC (2006) Synaptic integration of olfactory information in mouse anterior olfactory nucleus. J Neurosci 26:12023-12032.

Litaudon P, Amat C, Bertrand B, Vigouroux M, Buonviso N (2003) Piriform cortex functional heterogeneity revealed by cellular responses to odours. Eur J Neurosci 17:2457-2461.

Luskin MB, Price JL (1982) The distribution of axon collaterals from the olfactory bulb and the nucleus of the horizontal limb of the diagonal band to the olfactory cortex, demonstrated by double retrograde labeling techniques. J Comp Neurol 209:249-263.

Maarse H (1991) Volatile compounds in foods and beverages. New York: Dekker.

Menager I, Jost M, Aubert C (2004) Changes in physicochemical characteristics and volatile constituents of strawberry (Cv. Cigaline) during maturation. J Agric Food Chem 52:1248-1254.

Mori K, Takahashi YK, Igarashi KM, Yamaguchi M (2006) Maps of odorant molecular features in the mammalian olfactory bulb. Physiol Rev 86:409-433.

Murakami M, Kashiwadani H, Kirino Y, Mori K (2005) State-dependent sensory gating in olfactory cortex. Neuron 46:285-296.

Neville KR, Haberly LB (2004) Olfactory cortex. In: The synaptic organization of the brain, Ed 5 (Shepherd GM, ed), pp 415-454. New York: Oxford UP.

Penn DJ, Oberzaucher E, Grammer K, Fischer G, Soini HA, Wiesler D, Novotny MV, Dixon SJ, Xu Y, Brereton RG (2007) Individual and gender fingerprints in human body odour. J R Soc Interface 4:331-340.

Rennaker RL, Chen CF, Ruyle AM, Sloan AM, Wilson DA (2007) Spatial and temporal distribution of odorant-evoked activity in the piriform cortex. J Neurosci 27:1534-1542.

Scott JW, McBride RL, Schneider SP (1980) The organization of projections from the olfactory bulb to the piriform cortex and olfactory tubercle in the rat. J Comp Neurol 194:519-534.

Shepherd GM (2006) Smell images and the flavour system in the human brain. Nature 444:316-321.

Shepherd GM, Chen WR, Greer CA (2004) Olfactory bulb. In: The synaptic organization of the brain, Ed 5 (Shepherd GM, ed), pp 165-216. New York: Oxford UP.

Skeen LC, Hall WC (1977) Efferent projections of the main and the accessory olfactory bulb in the tree shrew (Tupaia glis). J Comp Neurol 172:1-35.

Takahashi YK, Kurosaki M, Hirono S, Mori K (2004) Topographic representation of odorant molecular features in the rat olfactory bulb. J Neurophysiol 92:2413-2427.

The Netherlands Organization for Applied Scientific Research (2007) Volatile compounds in Food 9.1. http://www.vcf-online.nl/VcfHome.cfm. Zeist, The Netherlands: TNO.

Wilson DA (1998) Habituation of odor responses in the rat anterior piriform cortex. J Neurophysiol 79:1425-1440.

Wilson DA (2001) Receptive fields in the rat piriform cortex. Chem Senses 26:577-584.

Wilson DA (2003) Rapid, experience-induced enhancement in odorant discrimination by anterior piriform cortex neurons. J Neurophysiol 90:65-72.

Zou Z, Buck LB (2006) Combinatorial effects of odorant mixes in olfactory cortex. Science 311:1477-1481.

Zou Z, Horowitz LF, Montmayeur JP, Snapper S, Buck LB (2001) Genetic tracing reveals a stereotyped sensory map in the olfactory cortex. Nature 414:173-179.

Zou Z, Li F, Buck LB (2005) Odor maps in the olfactory cortex. Proc Natl Acad Sci USA 102:7724-7729. 